\title{
Uranyl Ion Complexes with Long-Chain Aliphatic $\alpha, \omega$-Dicarboxylates and 3d-Block Metal Counterions
}

Pierre Thuéry and Jack Harrowfield

Crystal structure refinement details

p. S2

Additional structural figures

p. S5

Emission spectra

p. S8

Infrared spectra

p. $\mathrm{S} 10$ 


\section{Crystal structure refinement details}

Compound 2. Atom U2 is disordered over two positions close to one another and related by an inversion centre, and it has been refined with half-occupancy accordingly. The atoms of the coordination sphere of $\mathrm{U} 2$, as well as part of the aliphatic chain of the dicarboxylate ligand bound to it, are also disordered and could only be modeled with restraints on bond lengths, angles and displacement parameters. The water solvent molecules have been given 0.5 occupancy factors in order to retain acceptable displacement parameters and so as to account for some too close contacts between them, and their hydrogen atoms were neither found, nor introduced. The structure was refined as corresponding to a 2component twin.

Compounds 3 and 4. Atom C6 is disordered over two positions which were refined with occupancy parameters constrained to sum to unity. Other parts of the dicarboxylate ligands display large and very anisotropic displacement parameters which probably indicate unresolved disorder. Restraints on some bond lengths, angles and displacement parameters had to be applied in these parts. The hydrogen atoms of the water molecule were neither found, nor introduced.

Compound 5. Part of one aliphatic chain (C23 and C24) is disordered over two positions which were refined with occupancy parameters constrained to sum to unity and restraints on bond lengths, angles and displacement parameters. The hydrogen atoms of the water molecule were neither found, nor introduced.

Compound 6. One dicarboxylate ligand (containing $\mathrm{O} 7$ to $\mathrm{O} 10$ ) is disordered around an inversion centre; one complete molecule has been refined with restraints on bond lengths, angles and displacement parameters.

Compound 7. Restraints on bond lengths, angles and/or displacement parameters had to be applied for one uranyl cation (U2) and several parts of the aliphatic chains. Two water 
solvent molecules were given occupancy factors of 0.5 in order to retain acceptable displacement parameters. The hydrogen atoms of water molecules were neither found, nor introduced. The highest residual electron density peaks are located near the uranium atoms, possibly as a result of imperfect absorption corrections and/or unresolved disorder.

Compound 8. Four water solvent molecules were given occupancy factors of 0.5 so as to retain acceptable displacement parameters and/or to account for too close contacts between them. Restraints on displacement parameters were applied for three water solvent molecules. The hydrogen atoms of water molecules were neither found, nor introduced. PLATON/TwinRotMat ${ }^{1}$ suggests possible twinning, but taking it into account did not result in any improvement of the refinement.

Compound 9. The highly anisotropic displacement parameter of atom C12 indicates that unresolved disorder of part of the corresponding aliphatic chain may be present. The highest residual electron density peaks are located near the uranium atoms, possibly as a result of imperfect absorption corrections.

Compound 10. The highest residual electron density peaks are located near the uranium atoms, possibly as a result of imperfect absorption corrections.

Compound 11. Part of the dicarboxylate ligand (atoms $\mathrm{O} 3, \mathrm{O} 4$ and $\mathrm{C} 1$ to $\mathrm{C} 6$ ) is disordered over two positions which were refined with occupancy parameters constrained to sum to unity and restraints on bond lengths, angles and displacement parameters. The highest negative residual electron density peak is located near the uranium atom, possibly as a result of imperfect absorption corrections.

Compound 12. Atom U3 is disordered over two positions that are both close to an inversion centre, and that have been given occupancy parameters of 0.25 accordingly; the disorder of the oxygen atoms bound to it was only partly resolved, with two positions being found for the oxo atom $\mathrm{O} 5$ and the carboxylate atom $\mathrm{O} 20$, but not for the other carboxylate 
(O19) and the water molecule (O23). Restraints on bond lengths and angles were applied for the disordered uranyl moiety and restraints on displacement parameters were applied for the disordered parts and some too strongly anisotropic carbon atoms in the aliphatic chains.

1 Spek, A. L. J. Appl. Crystallogr. 2003, 36, 7-13. 
Figure S1. View of complex 4. Displacement ellipsoids are drawn at the $40 \%$ probability level. Symmetry codes: $\mathrm{i}=-x-1,1-y, 2-z ; \mathrm{j}=x+1, y-1, z ; \mathrm{k}=-x,-y, 2-z ; 1=x-1, y$, $z ; \mathrm{m}=x-1, y+1, z ; \mathrm{n}=x+1, y, z$. Only one position of the disordered part is represented; counterions, solvent molecules and hydrogen atoms are omitted.

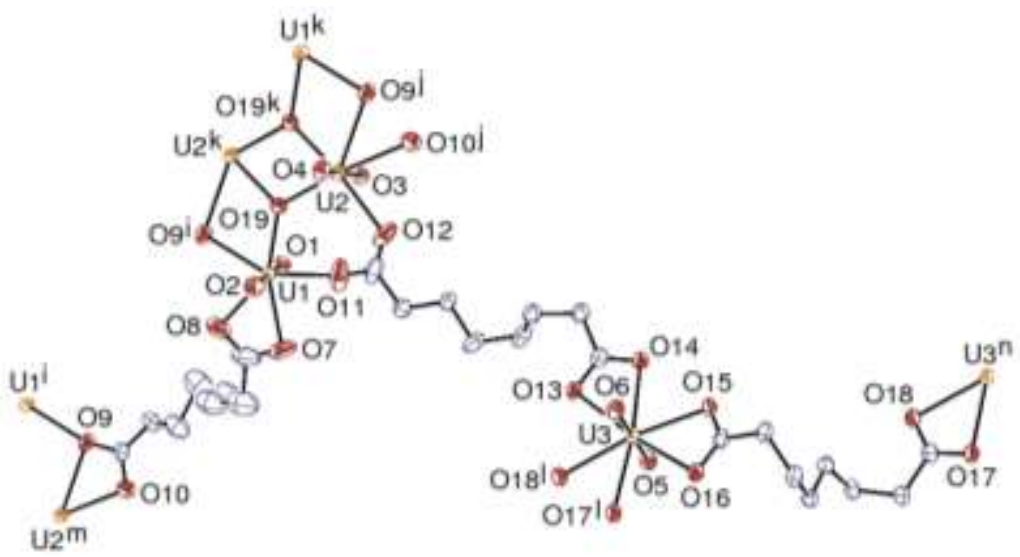

Figure S2. Top: The 2D assembly in complex 5 viewed edge-on. Bottom: View of the packing with layers viewed edge-on. The uranium coordination polyhedra are colored yellow and those of cobalt orange. Solvent molecules and hydrogen atoms are omitted.
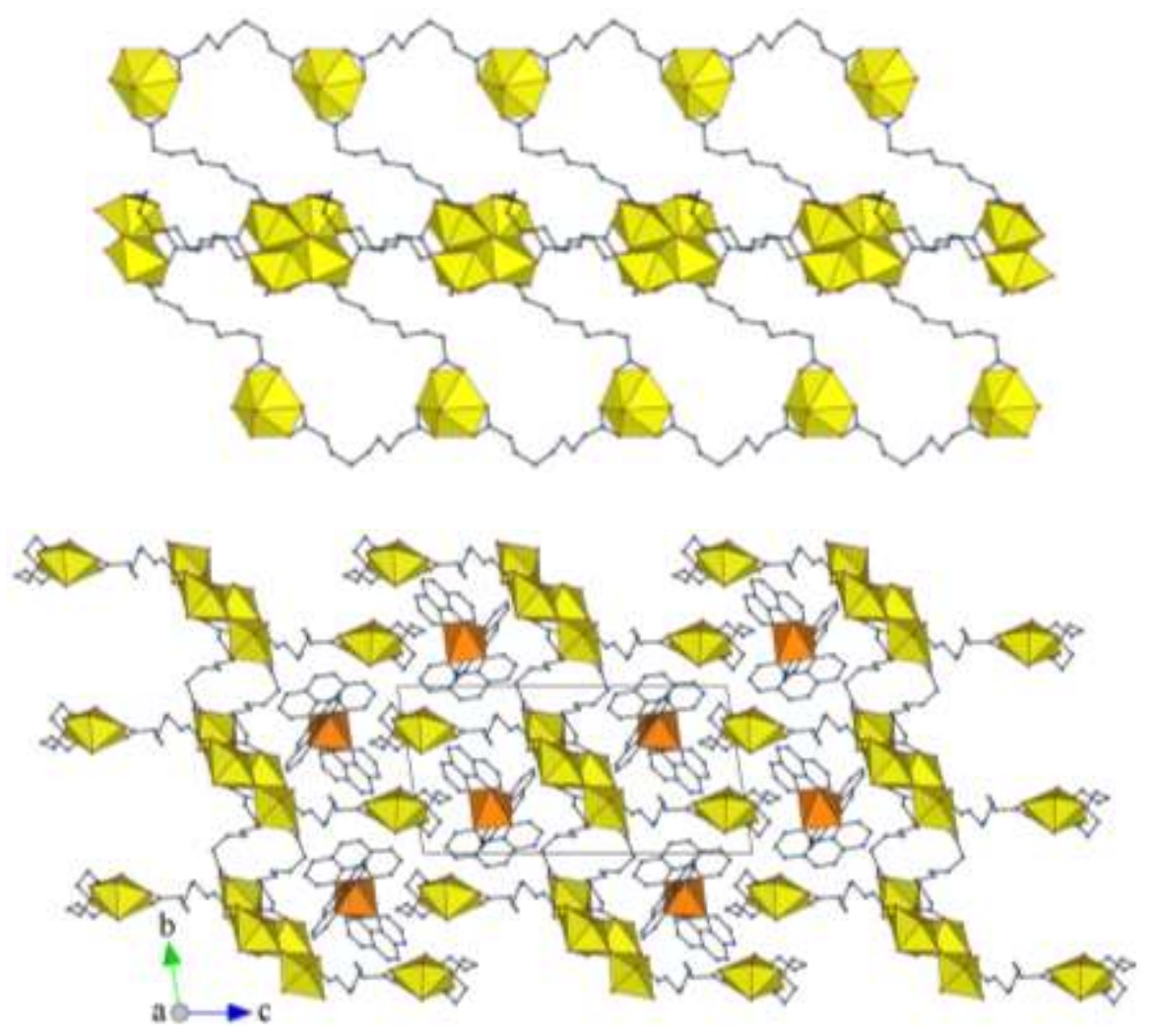
Figure S3. View of complex 9. Displacement ellipsoids are drawn at the 50\% probability level. Symmetry codes: $\mathrm{i}=1-x, y-1 / 2,1 / 2-z ; \mathrm{j}=-x, y+1 / 2,5 / 2-z ; \mathrm{k}=1-x, y+1 / 2,1 / 2$ $-z ; 1=-x, y-1 / 2,5 / 2-z$. Solvent molecules and hydrogen atoms are omitted.

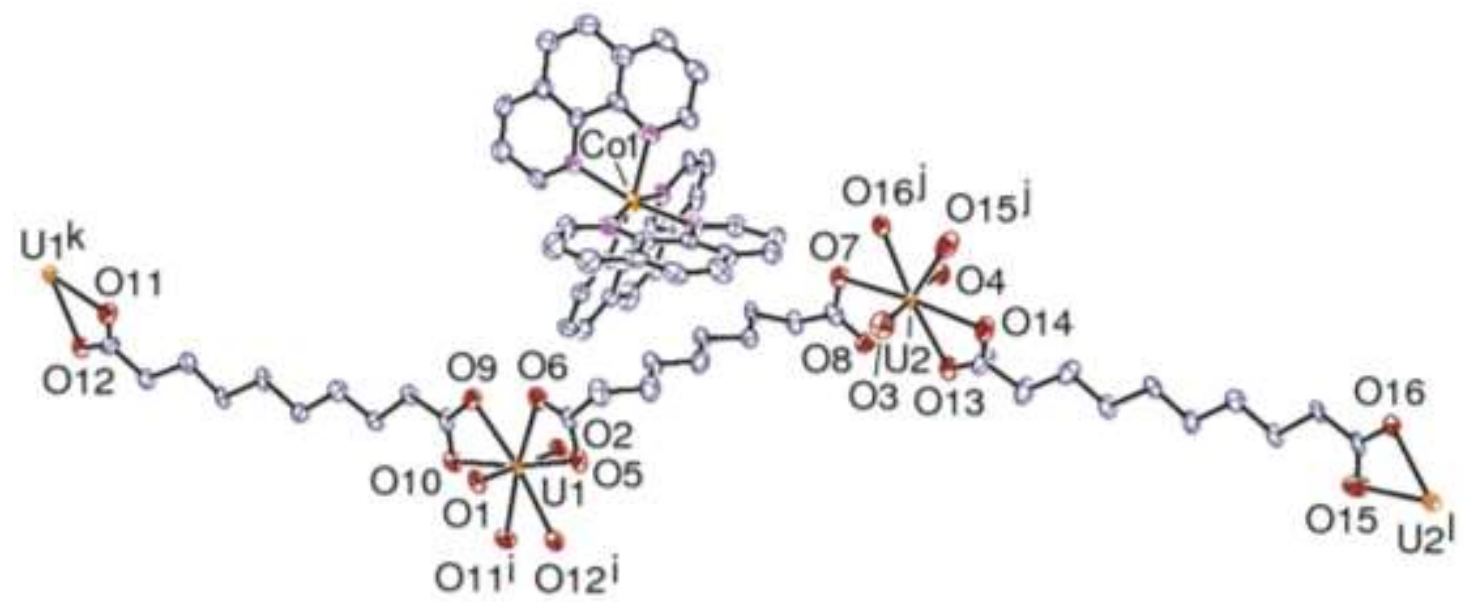


Figure S4. Top: View of complex 10. Displacement ellipsoids are drawn at the 50\% probability level. Symmetry codes: $\mathrm{i}=1-x, y-1 / 2,1 / 2-z ; \mathrm{j}=-x, y+1 / 2,5 / 2-z ; \mathrm{k}=1-x$, $y+1 / 2,1 / 2-z ; 1=-x, y-1 / 2,5 / 2-z$. Middle: Two views of the $2 \mathrm{D}$ assembly. Bottom: Packing with the uranium coordination polyhedra yellow and those of nickel green. Solvent molecules and hydrogen atoms are omitted.
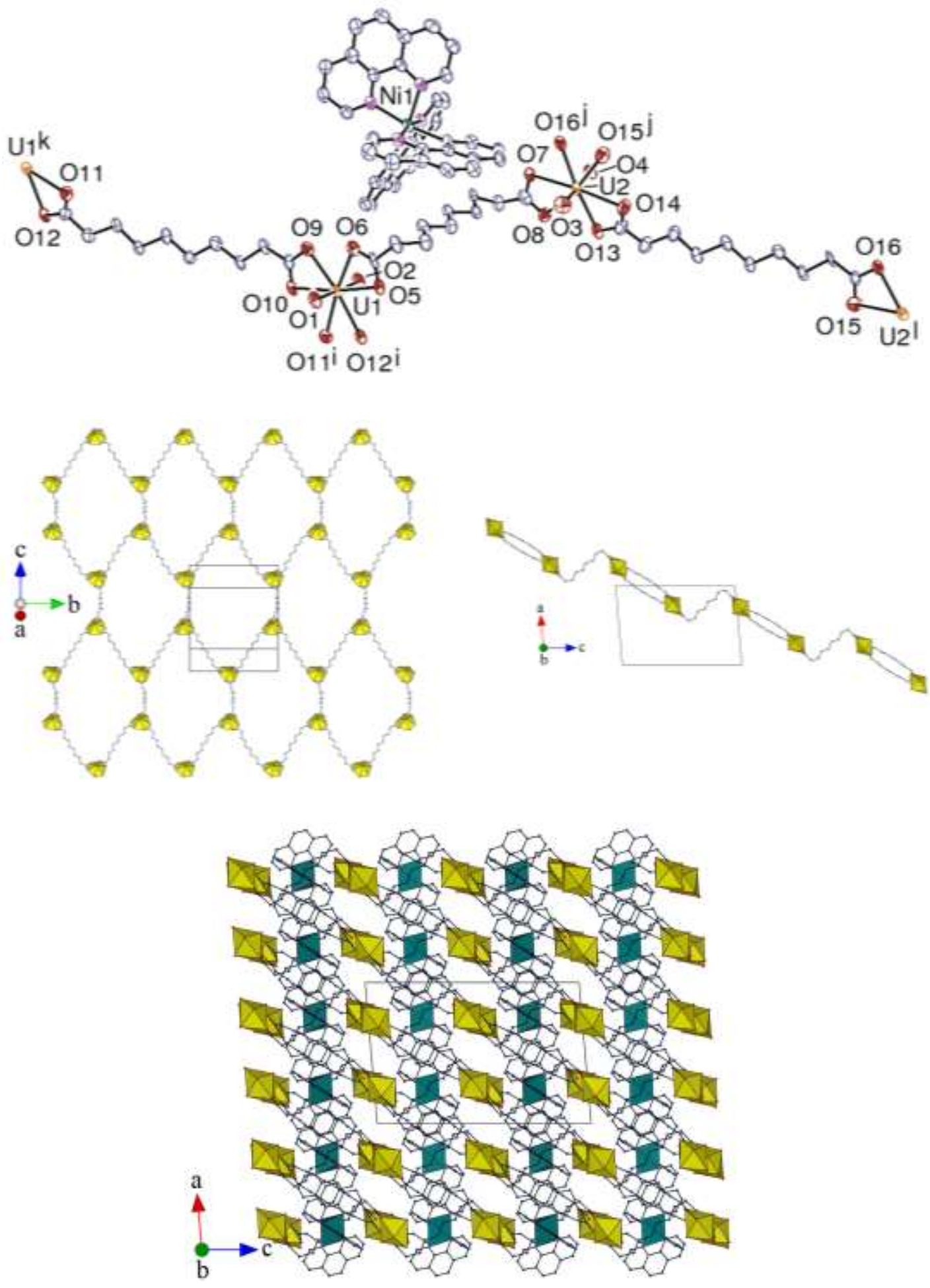
Figure S5. Solid state emission spectra of complexes 1, 3-10 and 12. Intensity (a. u.) is given versus wavelength $(\mathrm{nm})$. Excitation wavelength $420 \mathrm{~nm}$.
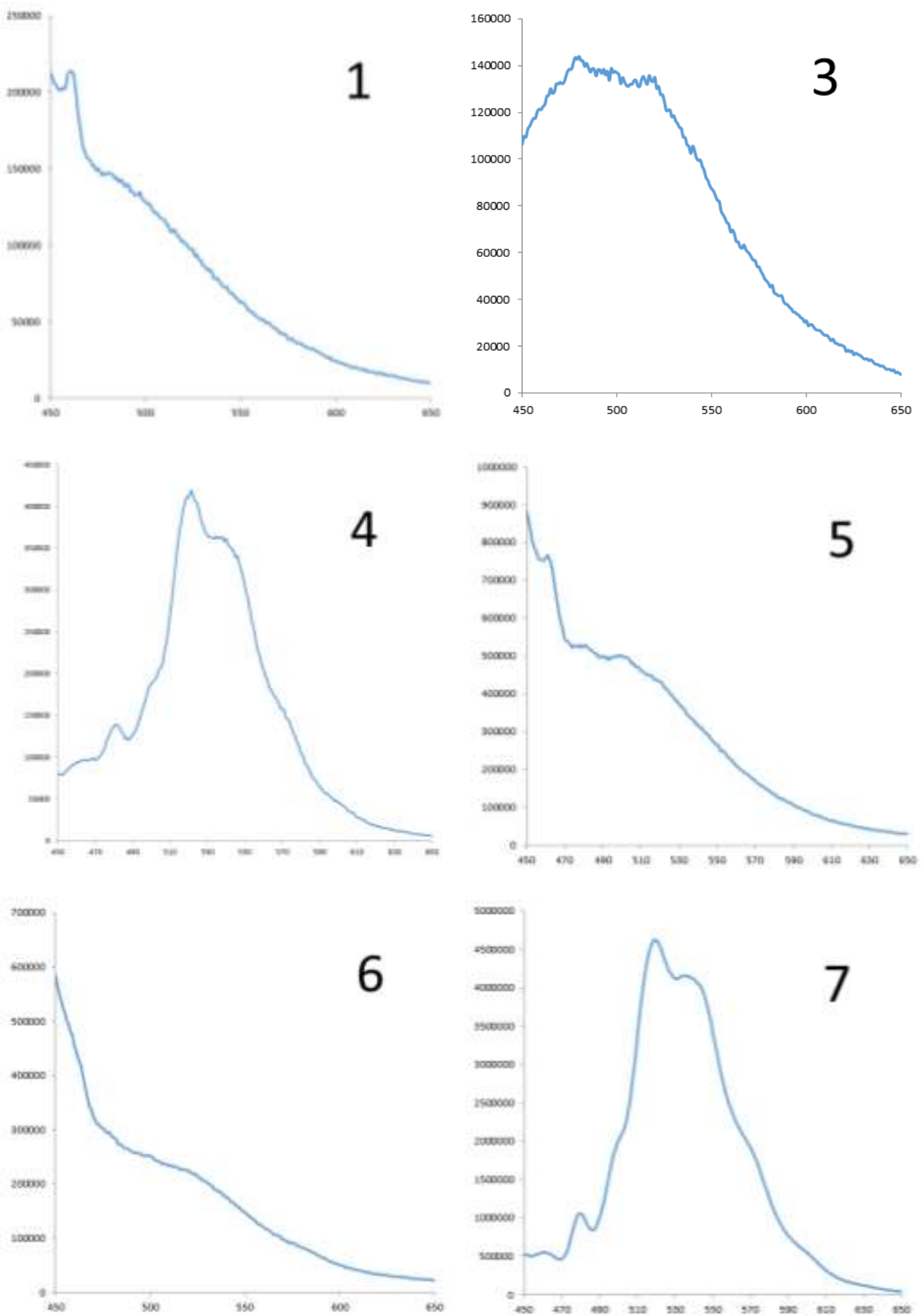
Figure S5 (Contnd.)
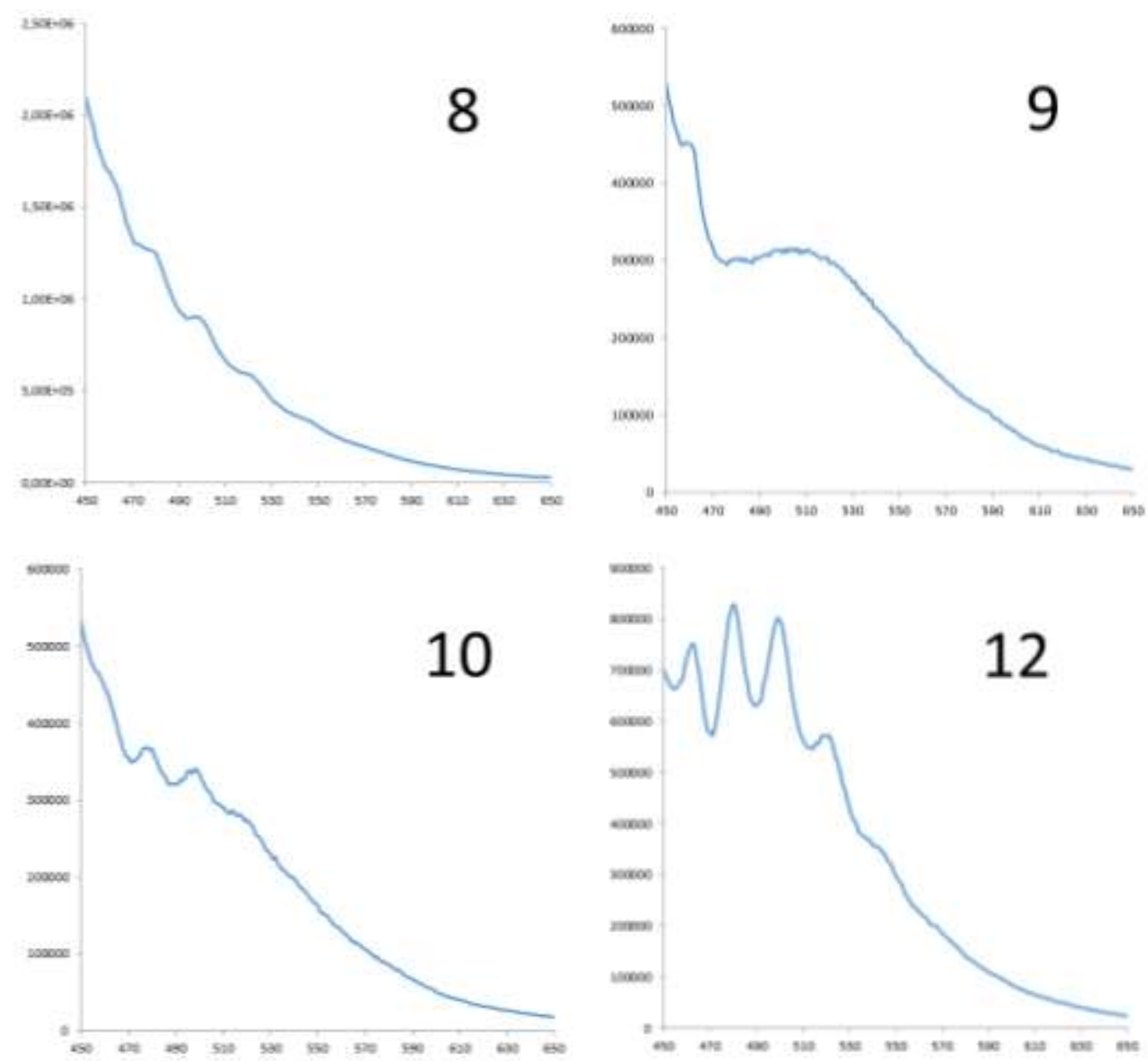
Figure S6. Infrared spectra (except for 6, which was obtained in very low yield).

1

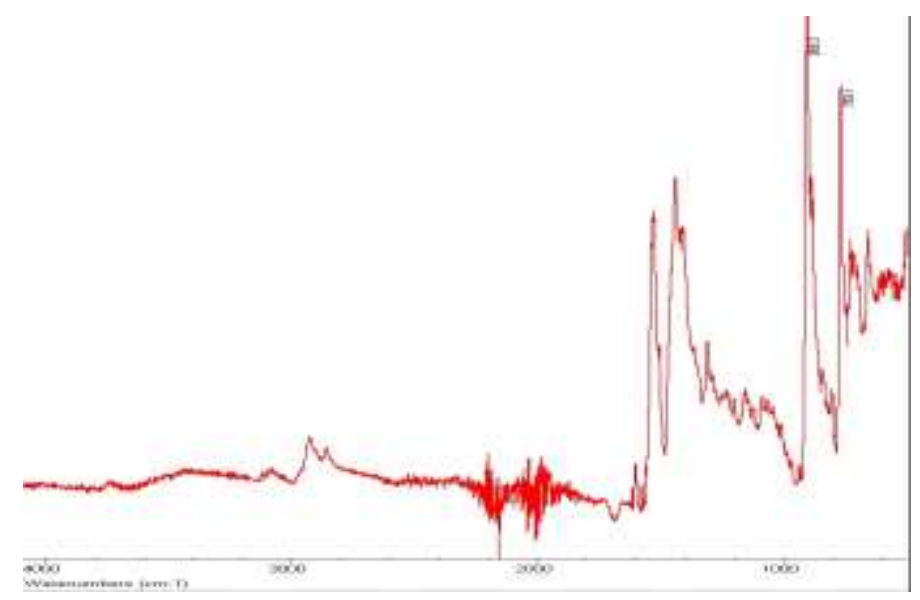

2

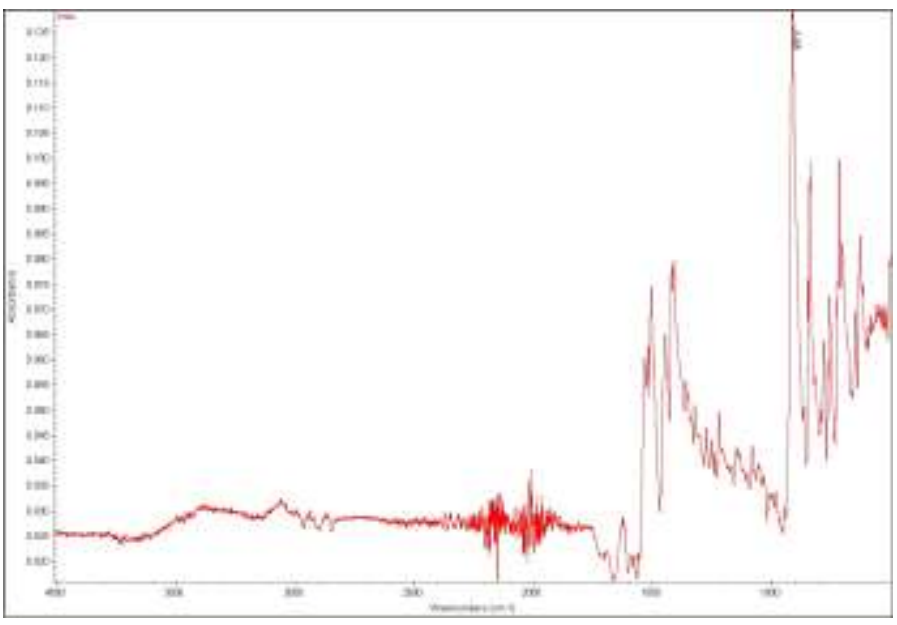

3

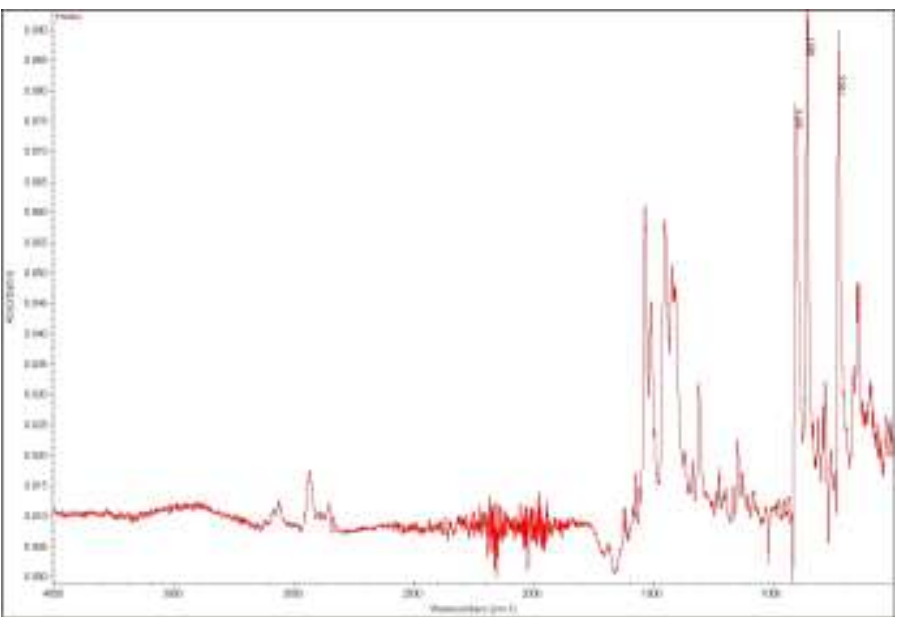




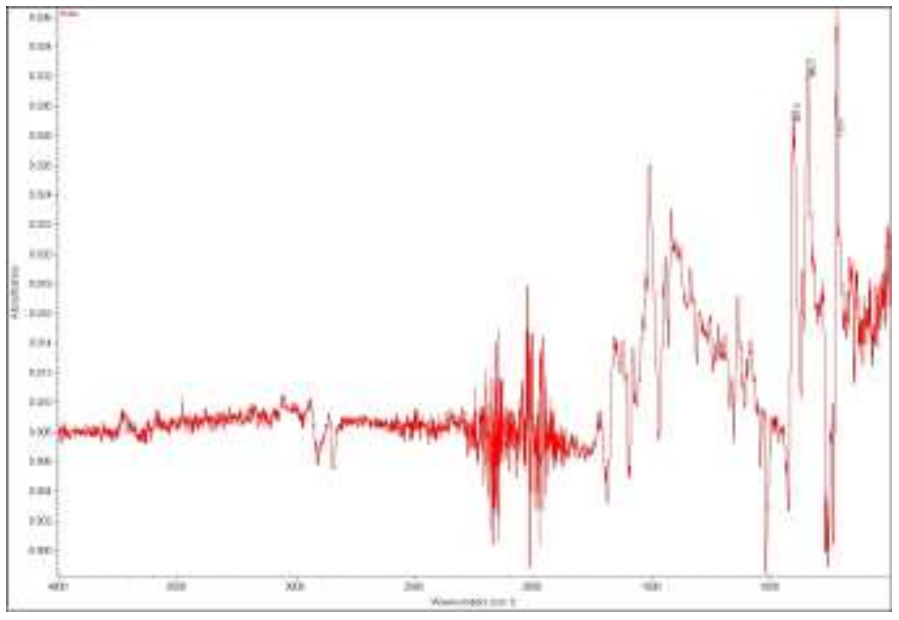

5

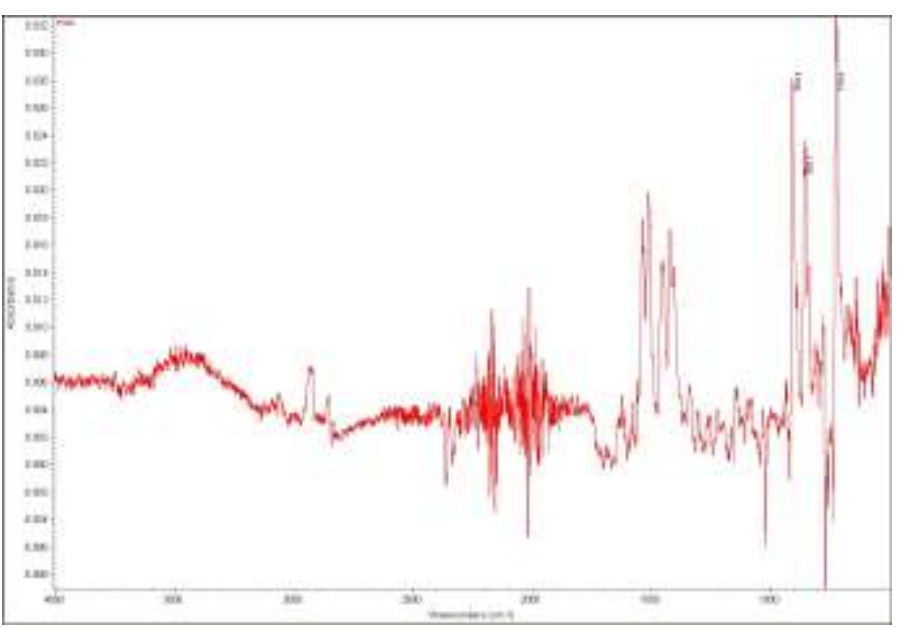

7

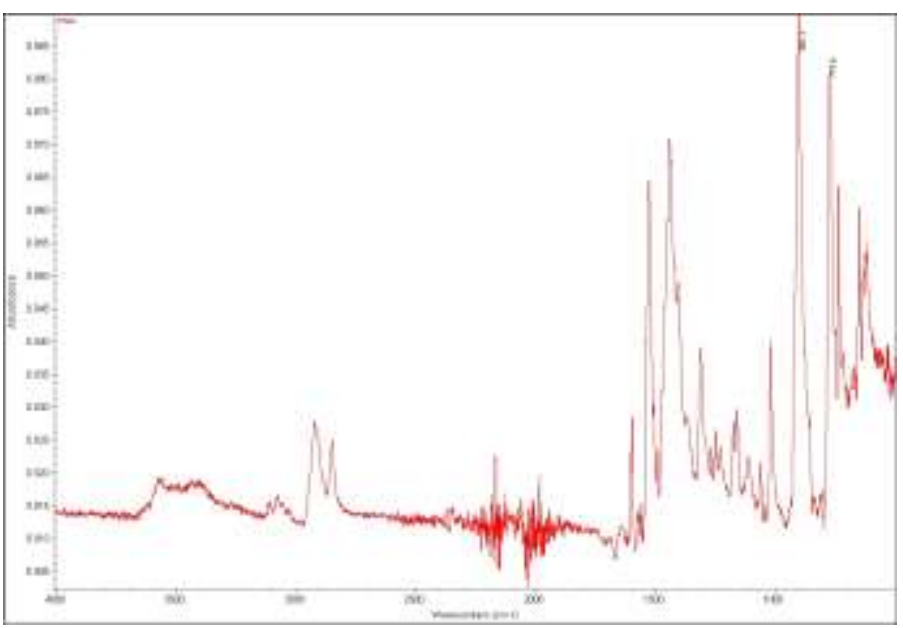


8

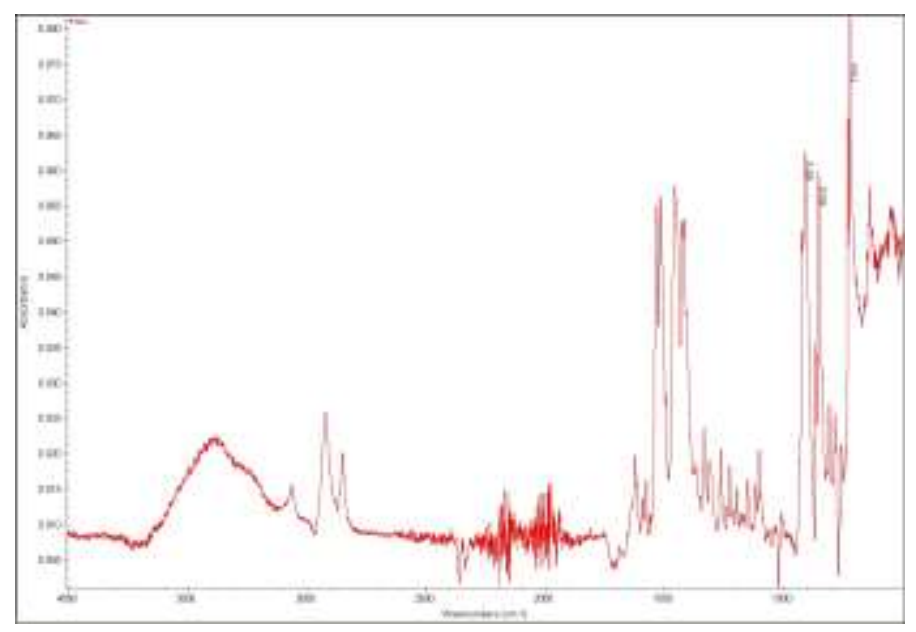

9

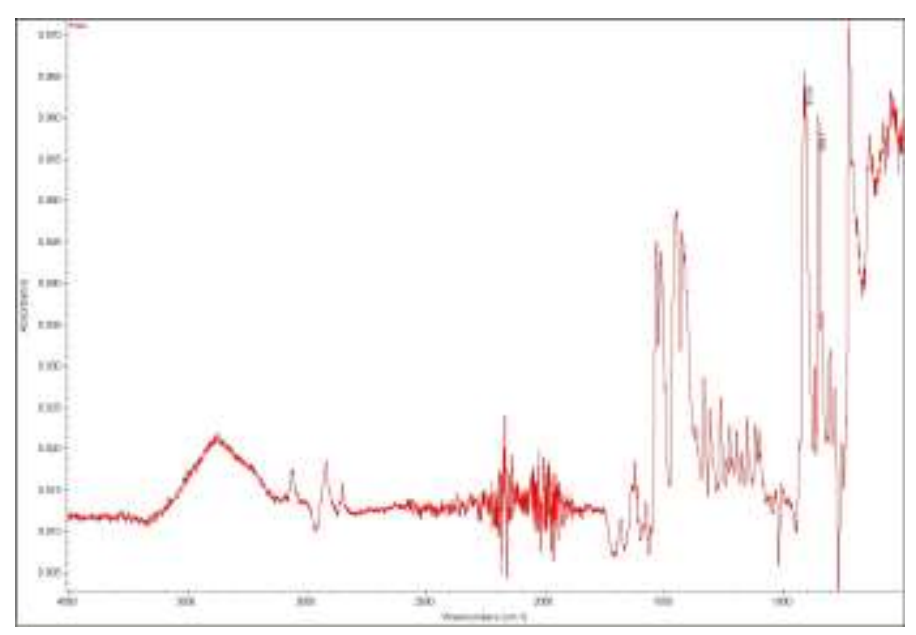

10

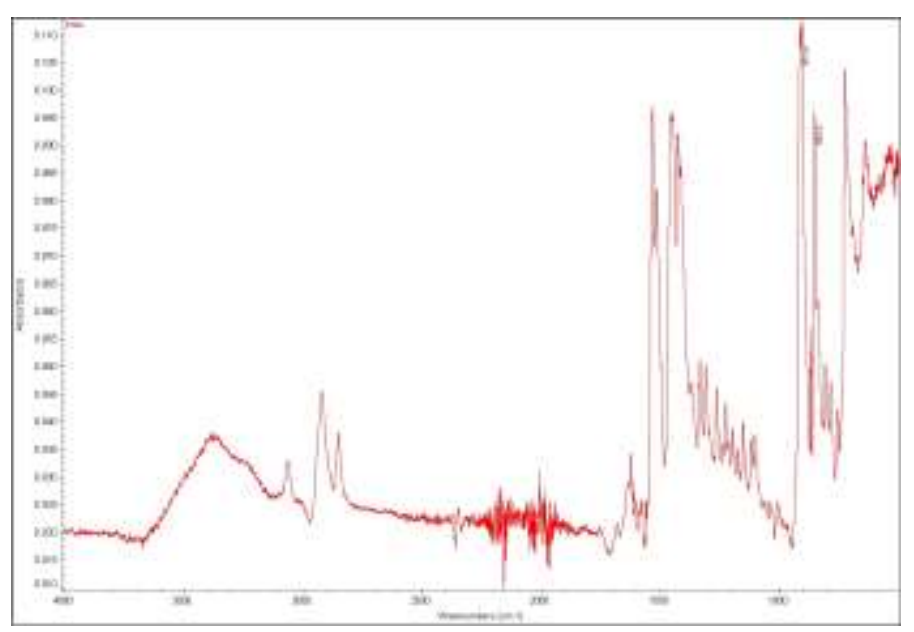

S12 


\section{1}

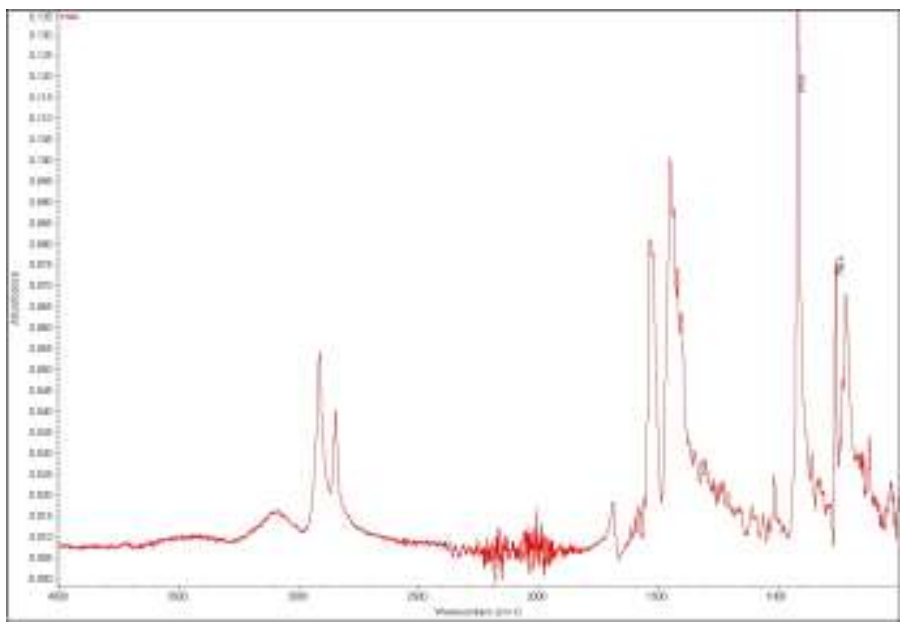

12

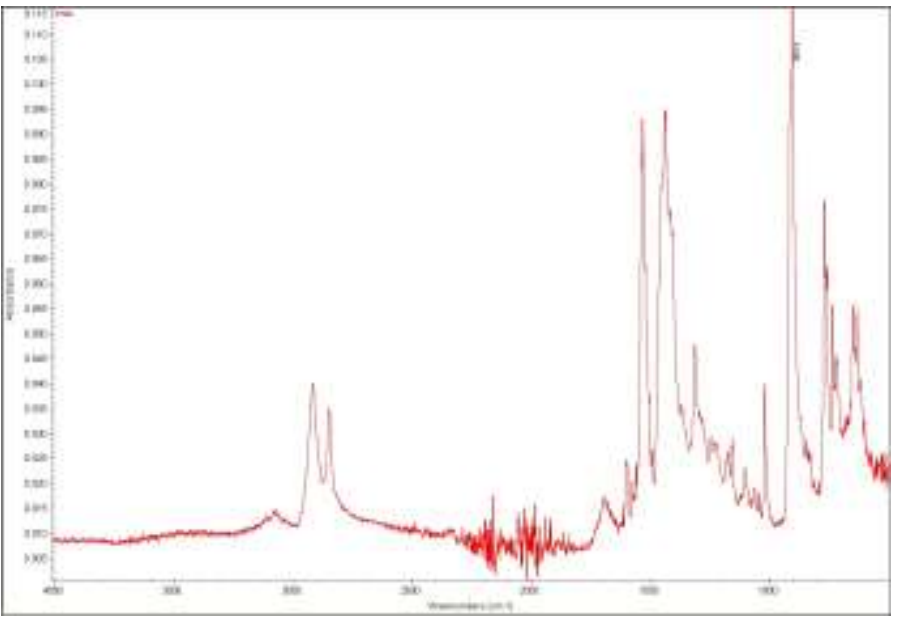

Frequencies of the uranyl stretching modes:

$\mathbf{1}: 907 \mathrm{~cm}^{-1} \quad \mathbf{2}: 908 \mathrm{~cm}^{-1}$

3: $908 \mathrm{~cm}^{-1} \quad \mathbf{4}: 908 \mathrm{~cm}^{-1}$

$5: 904 \mathrm{~cm}^{-1} \quad \mathbf{7}: 901 \mathrm{~cm}^{-1}$

$\mathbf{8}: 908 \mathrm{~cm}^{-1} \quad \mathbf{9}: 911 \mathrm{~cm}^{-1}$

$\mathbf{1 0}: 901 \mathrm{~cm}^{-1} \quad \mathbf{1 1}: 920 \mathrm{~cm}^{-1}$

$12: 904 \mathrm{~cm}^{-1}$. 